\title{
Vascular anastomosis time as a risk factor for delayed graft function in single renal transplantation from deceased donors
}

\author{
S. Dost ${ }^{1}$, B. Kunduzi ${ }^{1}$, I. Mosca ${ }^{2}$, A. Shamali ${ }^{2}$, J. Olsburgh², N. Karydis ${ }^{2}$ \\ ${ }^{1}$ King's College London, School of Medicine \\ ${ }^{2}$ Guy's Hospital, Department of Nephrology and Transplantation
}

\section{Introduction:}

Recent evidence suggests that prolonged vascular anastomosis time (AT) in deceased donor renal transplantation may adversely affect graft survival ${ }^{1}$, due to rapid kidney warming. The impact of AT on the incidence of delayed graft function (DGF), however, has only recently emerged as a potential modifiable factor in renal transplantation. We aim to elucidate the role of AT in relation to DGF in a single centre cohort of DBD and DCD renal transplants.

\section{Methods:}

Prospective data from 138 consecutive single renal transplants from deceased donors over a period of 18 months were analysed. Static cold storage of renal grafts was used in all cases. In our unit, DGF is defined as the need for dialysis within one week from transplantation. A number of donor and recipient variables were examined in association with the incidence of DGF. Among these, donor age, donor type (DBD, DCD), cold storage time (CST), quality of graft perfusion (good, fair), recipient age and AT were included in univariate and multivariate logistic regression analysis. Using ROC curve analysis, we defined an AT of $33.5 \mathrm{~min}$ as the cut-off value for "short" and "long" AT groups in our sample.

Results:

86 DBD and 52 DCD renal transplants with a respective $30.2 \%$ and $59.6 \%$ DGF rate $(p=0.001)$ were analysed (Table 1$)$. AT longer than 33.5 minutes, DCD donation, donor age and recipient age were significant risk factors for DGF in univariate analysis ( $p=0.008, \mathrm{OR} 4.08 ; p=0.001$, OR 3.4; $p=0.024, \mathrm{OR} 1.03$; and $p=0.025, \mathrm{OR} 1.03$, respectively). The quality of graft perfusion and cold storage time were not associated with DGF. In the multivariate model, DCD donation and long AT remained significant predictors of DGF ( $p=0.003$, OR 3.5 and $p=0.032$, OR 3.5, respectively) (Table 2). Interestingly, DBD grafts were more likely to develop DGF due to prolonged AT (OR 5.2, $p=0.037$ ) (Table 3), compared to DCD grafts.

\section{Discussion:}

Two recent reports on DBD renal transplants have shown a detrimental effect of prolonged AT on early graft function ${ }^{2,3}$, with an increased incidence of DGF. Our study included both DBD (62.3\%) and DCD (37.7\%) grafts. DCD donation remained an independent risk factor for DGF, however longer AT did not have a significant impact on the incidence of DGF in this subgroup (Table 3). Presumably, the higher DGF rate in DCD grafts (Table 1) may be attributed to the first warm ischemic time at the time of donation. On the other hand, long AT had a significant impact in the DBD subgroup, leading to a five-fold increase in the risk of DGF (Table 3). These findings are in accordance with recent reports and highlight AT as an important modifiable risk factor.

\section{Conclusion:}

AT longer than 33.5 minutes is a significant risk factor for DGF in single deceased donor renal transplants after cold storage. The development of surgical strategies to maintain a short AT would probably result in lower DGF rates with obvious benefits with regard to length of hospital stay and cost.

\begin{tabular}{|c|c|c|c|}
\hline & \multicolumn{2}{|c|}{ DGF } & \multirow[b]{2}{*}{$p$} \\
\hline & Yes & No & \\
\hline No of patients $(\mathrm{N}=138)$ & 57 & 81 & \\
\hline Donor age & $55.4 \pm 13.8$ & $49.0 \pm 17.0$ & 0.024 \\
\hline Donor type & & & 0.001 \\
\hline$D B D$ & 26 (30.2\%) & $60(69.8 \%)$ & \\
\hline$D C D$ & 31 (59.6\%) & $21(40.4 \%)$ & \\
\hline Recipient age & $54.2 \pm 12.0$ & $49.0 \pm 13.7$ & 0.025 \\
\hline Perfusion quality & & & 0.169 \\
\hline Good & $27(37.5 \%)$ & $45(62.5 \%)$ & \\
\hline Fair & $10(55.6 \%)$ & $8(44.4 \%)$ & \\
\hline Cold storage time (min) & $793.6 \pm 280.6$ & $790.6 \pm 310.3$ & 0.956 \\
\hline Anastomotic time (min) & $43.6 \pm 11.5$ & $39.8 \pm 10.7$ & 0.058 \\
\hline Anastomotic time group & & & 0.008 \\
\hline Short (<33.5 min) & 5 (17.9\%) & $23(82.1 \%)$ & \\
\hline Long ( $\geq 33.5 \mathrm{~min})$ & 47 (47\%) & $53(53 \%)$ & \\
\hline
\end{tabular}

\begin{tabular}{|c|c|c|c|c|}
\hline & OR & 95\% Cl - Lower & $95 \% \mathrm{Cl}$ - Upper & $p$ \\
\hline Donor age & 0.997 & 0.965 & 1.030 & 0.867 \\
\hline Recipient age & 1.037 & 0.999 & 1.077 & 0.058 \\
\hline Donor type & 3.488 & 1.550 & 7.850 & 0.003 \\
\hline AT & 3.459 & 1.112 & 10.767 & 0.032 \\
\hline
\end{tabular}

\begin{tabular}{|c|c|c|c|c|}
\hline Donor type & OR & $95 \% \mathrm{Cl}$ - Lower & $95 \% \mathrm{Cl}$ - Upper & $p$ \\
\hline DBD & 5.2 & 1.1 & 24.6 & 0.037 \\
\hline DCD & 2.8 & 0.6 & 13.3 & 0.202 \\
\hline
\end{tabular}

\section{References:}

${ }^{1}$ Heylen L et al. The Impact of Anastomosis Time During Kidney Transplantation on Graft Loss: A Eurotransplant Cohort Study. AJT (2016)

2 Marzouk et al. The impact of vascular anastomosis time on early kidney transplant outcomes. Transplant Res (2013)

${ }^{3}$ Heylen et al. The effect of anastomosis time on outcome in recipients of kidneys donated after brain death: a cohort study. AJT (2015) 